\title{
Jia-Shen decoction-medicated serum inhibits angiotensin-II induced cardiac fibroblast proliferation via the TGF-ß1/Smad signaling pathway
}

\author{
LIN CUI $^{1 *}$, YOUPING WANG ${ }^{1 *}$, RUI YU $^{2 *}$, BIN LI $^{3}$, SHIYANG XIE ${ }^{1}$, \\ YUAN GAO $^{1}$, XIAOXIAO WANG ${ }^{1}$ and MINGJUN ZHU ${ }^{3}$ \\ ${ }^{1}$ Central Laboratory, First Affiliated Hospital, Henan University of Traditional Chinese Medicine; \\ ${ }^{2}$ Department of Internal Medicine, Henan University of Traditional Chinese Medicine; ${ }^{3}$ Division of Cardiology, \\ First Affiliated Hospital, Henan University of Traditional Chinese Medicine, Zhengzhou, Henan 450000, P.R. China
}

Received July 21, 2015; Accepted May 27, 2016

DOI: $10.3892 / \mathrm{mmr} .2016 .5405$

\begin{abstract}
Jia-Shen decoction (JSD) is a traditional Chinese medicine, which is used widely to treat chronic heart failure. However, the underlying mechanism remains to be elucidated. The present study aimed to investigate the mechanism underlying the effects of JSD on cardiac fibroblast $(\mathrm{CF})$ proliferation and differentiation. The CFs were obtained from the hearts of neonatal (1-3-day old) Sprague-Dawley rats and treated with JSD-medicated serum (JSDS) with or without angiotensin II (Ang II). Cell proliferation was assessed using Cell Counting Kit- 8 reagent. In addition, the mRNA expression levels of transforming growth factor- $\beta 1$ (TGF- $\beta 1)$ and phosphorylated small mothers against decapentaplegic ( $p$-Smad) $2 / 3$ and their protein expression levels were analyzed. CF proliferation was significantly increased in the Ang II-treated group, compared with the control group $(\mathrm{P}<0.05)$. The expression levels of collagen, $\alpha$-smooth muscle actin, TGF- $\beta 1$ and $p-S m a d 2 / 3$ were also increased in the Ang II-treated group $(\mathrm{P}<0.05)$. Following JSDS treatment, the increased levels of collagen and cell proliferation were inhibited, and the increased expression levels of $\mathrm{p}-\mathrm{Smad} 2$ and $\mathrm{p}-\mathrm{Smad} 3$ were also inhibited $(\mathrm{P}<0.05)$. These data suggested that JSDS may inhibit CF proliferation via attenuating the TGF- $\beta 1 /$ Smad signaling pathway.
\end{abstract}

Correspondence to: Professor Mingjun Zhu, Division of Cardiology, First Affiliated Hospital, Henan University of Traditional Chinese Medicine, 19 Renmin Road, Jinshui, Zhengzhou, Henan 450000, P.R. China

E-mail: henantcm@126.com

*Contributed equally

Key words: cardiac fibroblasts, fibrosis, Jia-Shen decoction medicated serum, mechanism

\section{Introduction}

Chronic heart failure (CHF) is the ultimate outcome for the majority of cardiovascular diseases. Myocardial fibrosis is a common pathological feature observed in numerous patients with heart disease, and is hypothesized to be the final common pathway, which ultimately results in irreversible organ failure (1). Cardiac fibroblasts (CFs) are one of the most important factors contributing to $\mathrm{CHF}$, which may be activated in response to hypertrophic stimuli, including angiotensin II (Ang II) (2-8). Ang II has been shown to be important in mediating cardiovascular diseases, and may be involved in the pathogenesis of heart failure (9-16).

Previously, a number of natural products have been used in the treatment of cardiovascular diseases, such as Qiliqiangxin, Osthole and Tanshinone IIA (17-22). Among them, Chinese medicine has been investigated extensively, and appears to be safe and effective. Jia-Shen decoction (JSD), a type of Chinese medicine, has been routinely used in treating patients with heart failure. Experimental investigation has shown that JSD can protect rats from hypoxia and hypothermia, and promote diuresis in rats with heart failure (23). It has also been used in the treatment of myocardial injury in animal models, with functions in reducing the size of myocardial infarction and improving cardiac function following MI (17). However, the molecular mechanism underlying the effect of JSD in myocardial protection remains to be fully elucidated.

The present study focused on the transforming growth factor (TGF)- $\beta 1 /$ small mothers against decapentaplegic (Smad) signaling pathway, and investigated whether JSD-medicated serum (JSDS) has a protective effect against Ang II-induced cardiac fibrosis.

\section{Materials and methods}

Ethics statement. All procedures in the present study were performed in accordance with the National Institute of Health Guide for the Care and Use of Laboratory Animals, which was also approved by the Care of Experimental Animals 
Committee of the Henan University of Traditional Chinese Medicine (Zhengzhou, China).

Isolation and culture of CFs. CFs were obtained from the hearts of neonatal (1-3-day-old) Sprague-Dawley rats (Henan Province Laboratory Animal Center (Zhengzhou, China), as previously described (19,24-26). Briefly, following sacrifice with an overdose of ether anesthesia, 20 neonatal hearts were rapidly excised. The CFs were detached with collagenase II (Thermo Fisher Scientific, Inc., Thermo Fisher Scientific, Inc., Waltham, MA, USA)/trypsin (Solarbio, Beijing, China) for $5 \mathrm{~min}$ at $37^{\circ} \mathrm{C}$ for 5-6 digestion periods. The collected cells were stained with trypan blue dye. cell suspension (90 $\mu \mathrm{l}$ ) was mixed with $10 \mu \mathrm{l}$ of $0.4 \%$ trypan blue dye and observed under a light microscope. Trypan blue-excluding cells were plated in petri dishes at a density of $5 \times 10^{6}$ cells $/ \mathrm{ml}$. The cultivation samples were incubated at $37^{\circ} \mathrm{C}$ in an atmosphere containing $5 \% \mathrm{CO}_{2}$ in Dulbecco's modified Eagle's medium (DMEM; Solarbio) supplemented with $10 \%$ bovine serum albumin (Solarbio). The culture medium was replaced 90 min following plating to remove myocytes, which did not attach to the dish. The identification of CFs was performed using immunofluorescence staining with anti-vimentin for fibroblasts. CFs at $80 \%$ confluence in the culture wells were digested using $0.25 \%$ trypsin and then passaged at 1:2 dilutions. Cells from the second to fourth passage were seeded at a density of $1 \times 10^{6}$ cells $/ \mathrm{ml}$ were utilized for the subsequent experiments.

The cells were incubated at $37^{\circ} \mathrm{C}$ in an atmosphere containing $5 \% \mathrm{CO}_{2}$ in serum-free DMEM supplemented with $0.1 \%$ bovine serum albumin for $24 \mathrm{~h}$, and then exposed to Ang II (Sigma-Aldrich, St. Louis, MO, USA) for another $24 \mathrm{~h}$. At the completion of the experiments, the cells were washed with cold phosphate-buffered saline (PBS) for subsequent experiments.

Preparation of JSDS. The JSDS was modified, according to a previous report (18). The 20 Sprague Dawley rats were randomly divided into a JSD group $(n=10)$ and a control group $(n=10)$. The rats were housed in a $20-25^{\circ} \mathrm{C}$ air-conditioned room with a 12-h light-dark cycle and provided standard chow with free access to tap water. The rats in the JSD group were treated by intragastric administration of JSD $(2.1 \mathrm{~g} / \mathrm{kg})$ three times a day for 3 days. The rats in the control group received intragastric administration of physiological saline twice a day for 3 days. At $1 \mathrm{~h}$ following the final administration, the rats were anesthetized using chloral hydrate intraperitoneally. Rats were sacrificed by an intraperitoneal injection of an overdose of chloral hydrate. Blood samples $(10 \mathrm{ml})$ were then collected from the abdominal aorta and centrifuged at $1,077 \mathrm{x} g$ at room temperature for $15 \mathrm{~min}$. The serum was isolated and stored at $-80^{\circ} \mathrm{C}$ until further analysis.

Cell proliferation assay and measurement of hydroxyproline. The cell suspension of CFs was inoculated into a 96-well plate at a density of $1 \times 10^{4}$ cells $/ \mathrm{ml}$. The CFs were divided into five groups: Control group, Ang II-treated group, Ang II with 2.5\% JSDS group, Ang II with 5\% JSDS group, and Ang II with $10 \%$ JSDS group. Following treatment, cell proliferation was assessed using Cell Counting Kit-8 (CCK8)
Table I. Sequences of the primers used in reverse transcription-quantitative polymerase chain reaction analysis.

\begin{tabular}{lc}
\hline Primer & \multicolumn{1}{c}{ Primer sequence (5'-3') } \\
\hline Gapdh-F & ACAGCAACAGGGTGGTGGAC \\
Gapdh-R & TTTGAGGGTGCAGCGAACTT \\
TGF- $\beta 1-F$ & CTGCTGACCCCCACTGATAC \\
TGF- $\beta 1-R$ & AGCCCTGTATTCCGTCTCCT \\
Colla1-F & GACTGTCCCAACCCCCAAAA \\
Colla1-R & TGGGTCCCTCGACTCCTATG \\
$\alpha-S M A-F$ & GGAGATGGCGTGACTCACAA \\
$\alpha-S M A-R$ & CGCTCAGCAGTAGTCACGAA
\end{tabular}

TGF- $\beta 1$, transforming growth factor- $\beta 1$; Colla1, collagen type I; $\alpha$-SMA, $\alpha$-smooth muscle actin; F, forward; R, reverse.

reagent (Dojindo Laboratories, Kumamoto, Japan) and cell culture medium was collected for measuring hydroxyproline content, according to the manufacturer's protocol. Cell culture medium was collected for measuring hydroxyproline content, according to the manufacturer's protocol (Nanjing Jiancheng Technology, Inc., Nanjing, China). The absorbance values (A450) were measured to evaluate cell numbers and estimate cell proliferation.

Total RNA extraction and reverse transcription-quantitative polymerase chain reaction $(R T-q P C R)$ analysis for $T G F-\beta 1$ $\alpha$-smooth muscle actin (SMA) and collagen I. The cell suspension of CFs was inoculated into 6-well plates at a density of $5 \times 10^{5}$ cells $/ \mathrm{ml}$. The CFs were divided into the groups: Control group, Ang II-treated group, and Ang II with $10 \%$ JSD group. Following treatment, the mRNA expression levels of $T G F-\beta 1, \alpha-S M A$ and collagen I were assayed using RT-qPCR analysis. Total RNA was isolated by extraction with TRIzol reagent (Invitrogen; Thermo Fisher Scientific, Inc.), according to the manufacturer's protocol. First strand cDNA was synthesized from $1 \mu \mathrm{g}$ of total RNA using the cDNA Synthesis kit (Invitrogen; Thermo Fisher Scientific, Inc.). qPCR was performed using a $20 \mu \mathrm{l}$ reaction mixture that contained $10 \mu \mathrm{l}$ SYBR Green supermix (Invitrogen; Thermo Fisher Scientific, Inc.), and $1 \mu \mathrm{g}$ cDNA with $0.5 \mathrm{mM}$ of each of the forward and reverse primer. The qPCR conditions were as follows: $95^{\circ} \mathrm{C}$ for preheating for $10 \mathrm{~min}$, and then 40 cycles of $95^{\circ} \mathrm{C}$ for $15 \mathrm{sec}$ and $60^{\circ} \mathrm{C}$ for $1 \mathrm{~min}$. To confirm amplification specificity, each qPCR product was tested by melting curve analysis. The reaction was conducted using an Applied Biosystems 7300 Real-Time PCR system (Thermo Fisher Scientific, Inc). The relative expression of mRNA was calculated using the $2^{-\triangle \Delta \mathrm{Cq}}$ method $(27,28)$. GAPDH mRNA was used as internal standard, and each results was normalized to GAPDH. The sequences of all primers used in the present study are presented in Table I.

Western blot analyses for TGF- $\beta 1$ and $p$-Smad2/3. The cardiac fibroblasts grown on 6 -well plates were harvested with $200 \mu 1$ volume of ice-cold Pierce RIPA buffer (Thermo 


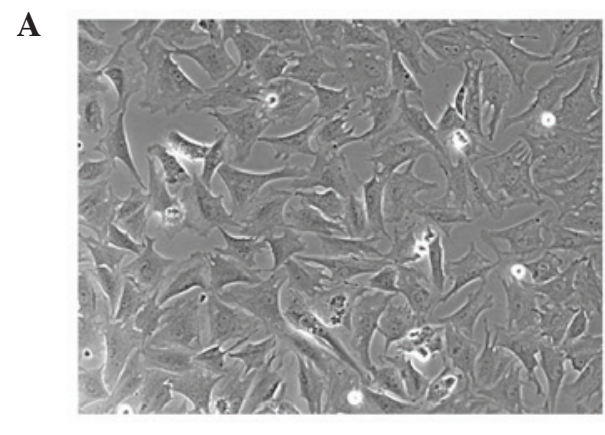

\section{B}

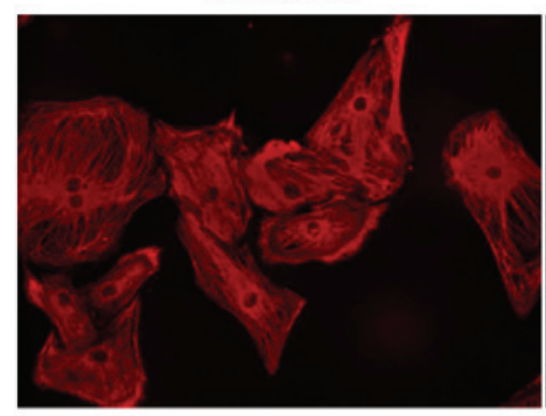

DAPI

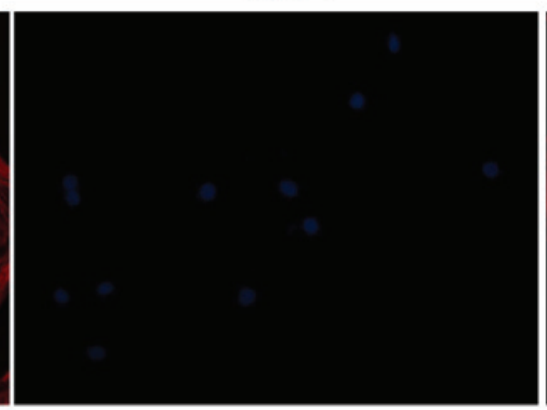

Merge

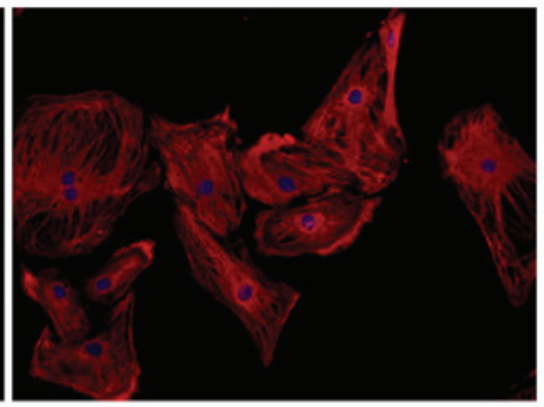

Figure 1. Characterization of primary cultured neonatal rat CFs. (A) Cell morphology of the first passage of neonatal rat CFs under an optical microscope. Original magnification, x20. (B) Primary cultured CFs were subjected to immunofluorescence staining for vimentin to determine their purity. Red shows vimentin staining; DAPI is shown in blue (nuclear). Original magnification, $\mathrm{x} 40$. CFs, cardiac fibroblasts.

Fisher Scientific, Inc.) supplemented with $10 \mu \mathrm{l} / \mathrm{ml}$ protease inhibitor cocktail (Sigma-Aldrich). Following centrifugation at $13,000 \mathrm{~g}$ for $30 \mathrm{~min}$ at $4^{\circ} \mathrm{C}$, the supernatants were collected and the total protein content was quantified using a Bradford protein assay. Subsequently, $50 \mu \mathrm{g}$ of the total protein extracts were electrophoresed on SDS-PAGE gels and blotted onto polyvinylidene difluoride membranes. The proteins were then blocked with $5 \%$ skimmed milk powder blocking solution for $60 \mathrm{~min}$, and incubated overnight at $4^{\circ} \mathrm{C}$ with rabbit polyclonal anti-TGF- $\beta 1$ (1:400; cat. no. ab92486; Abcam, Cambridge, UK), rabbit polyclonal anti-Smad2 (1:400; cat. no. AB3849; EMD Millipore, Billerica, MD, USA), rabbit monoclonal anti-p-Smad3 (1:400; cat. no. ab138659; Abcam), and mouse monoclonal anti-GAPDH (1:5,000; cat. no. G8795; Sigma-Aldrich) antibodies. Following incubation with a secondary horeseradish peroxidase-conjugated bovine anti-mouse (cat. no. sc-2380) and bovine anti-rabbit IgG (cat. no. sc-2370) (1:2,000; Santa Cruz Biotechnology, Inc., Santa Cruz, CA, USA), densitometric analysis of the bands was performed using Image J software 1.38 (National Institutes of Health, Bethesda, MD, USA), and the values were normalized with GAPDH protein.

Immunofluorescence assay for SMA, vimentin and collagen I. The cells grown on cover slips were fixed with $4 \%$ paraformaldehyde for $10 \mathrm{~min}$ at room temperature. Following permeabilization with $0.5 \%$ Triton-X100 for $10 \mathrm{~min}$, the fixed cells were blocked with $0.5 \%$ bovine serum albumin (Solarbio, Beijing, China) for $30 \mathrm{~min}$ and then incubated overnight at $4^{\circ} \mathrm{C}$ with the following primary antibodies: Mouse monoclonal anti-vimentin (1:500; cat. no. ab8978; Abcam), mouse monoclonal anti- $\alpha-\operatorname{SMA}(1: 500$; cat. no. BM0001 Boster Biological Technology, Wuhan, China) and rabbit polyclonal anti-type I collagen (1:200; cat. no. BM0325 Boster Biological Technology). The immunoreactions were revealed using specific anti-mouse fluorescein isothiocyanate-conjugated Affinipure Goat Anti-Mouse IgG (H+L) (1:200; cat. no. SA00003-1; Boster Biological Technology) and anti-rabbit Alexa Fluor 594-conjugated Goat Anti-Rabbit IgG Alexa Fluor-conjugated IgG (H+L) (1:200; cat. no. SA00006-4; Proteintech, Wuhan, China) for $1 \mathrm{~h}$ at room temperature. Negative controls were included by omitting the primary antibodies. Following washing with PBS, the immunolabeled cells were observed under a fluorescence microscope (Leica Microsystems, Mannheim, Germany). Densitometric analyses of the intensities of the vimintin, $\alpha$-SMA and collagen I fluorescent signals were performed on digitized images using Image J software.

Statistical analysis. All statistical analyses were performed using SPSS 18.0 software (SPSS, Inc., Chicago, IL, USA). All data are expressed as the mean \pm standard error of the mean. One-way analysis of variance was performed, with a post-hoc least significant difference test for comparisons among groups. $\mathrm{P}<0.05$ was considered to indicate a statistically significant difference.

\section{Results}

Characterization of primary cultured neonatal rat CFs. The first passage of neonatal rat CFs cultured in the present study had the typical morphological characteristics of fibroblasts, as evidenced by their spindle or polygonal shape and large ovoid nucleus (Fig. 1A). The results of the immunofluorescence staining revealed that these cells were positive for vimentin, a marker of fibroblasts (Fig. 1B). 
A

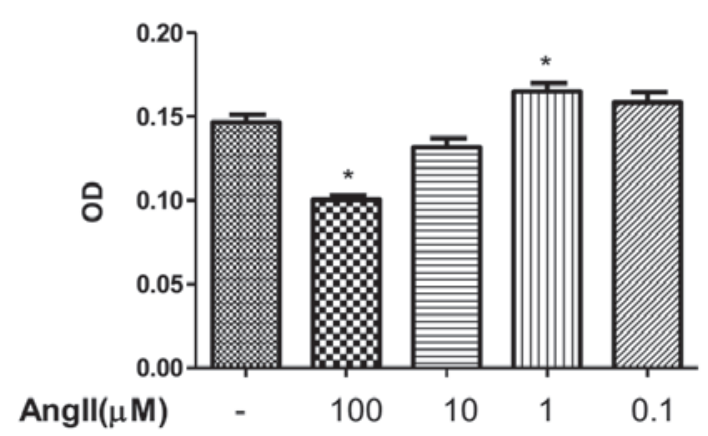

B

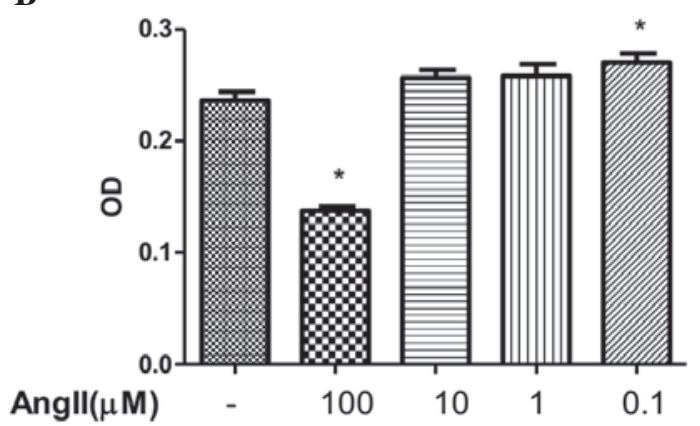

Figure 2. Effects of different concentrations of Ang II on cell proliferation in CFs. (A) Neonatal rat CFs were treated with different concentrations of Ang II for $24 \mathrm{~h}$. (B) CFs were treated with different concentration of Ang II for $48 \mathrm{~h}$. Data are presented as the mean \pm standard error of the mean. * $\mathrm{P}<0.05$, compared with the non-Ang II-treated group. CFs, cardiac fibroblasts; Ang II, angiotensin II; OD, optical density.

A

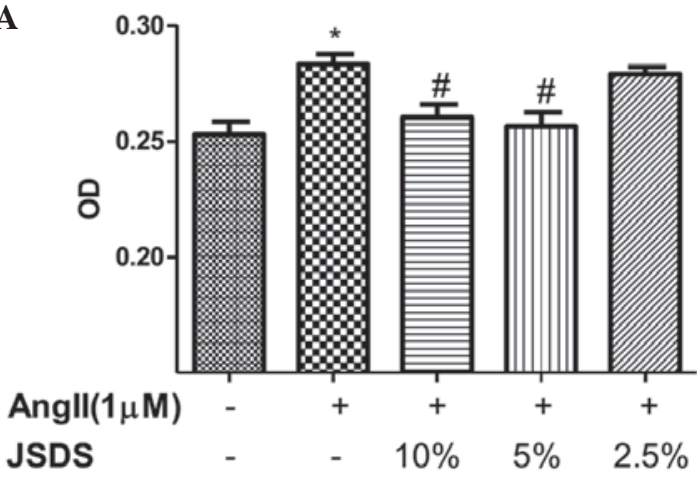

B

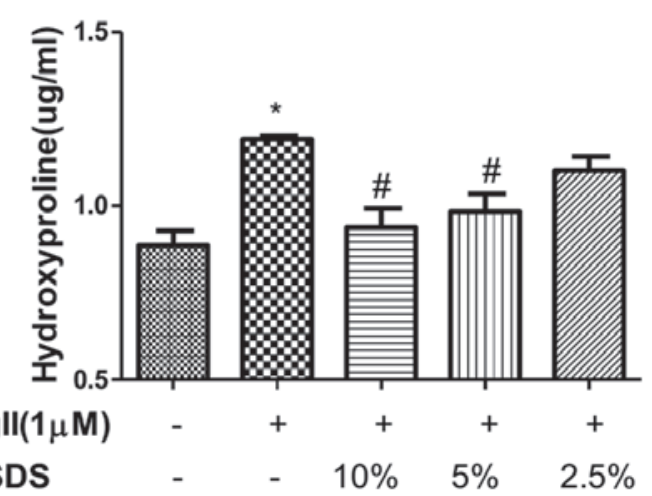

Figure 3. JSDS inhibits Ang II-induced cell proliferation and reduces collagen content in CFs. (A) Neonatal rat CFs were treated with different concentrations of JSDS and Ang II $(1 \mu \mathrm{M})$ for $24 \mathrm{~h}$. The number of cells are presented as an OD value, determined using a cell count assay. (B) Content of hydroxyproline in cell culture medium. ${ }^{*} \mathrm{P}<0.05$, compared with the control group; ${ }^{*} \mathrm{P}<0.05$, compared with the Ang II-treated group. Data are presented as the mean \pm standard error of the mean. CFs, cardiac fibroblasts; JSDS, Jia-Shen decoction-medicated serum; Ang II, angiotensin II; OD, optical density.

JSDS inhibits Ang II-induced CF proliferation and collagen content. To determine whether JSDS inhibited CF proliferation, the neonatal rat $\mathrm{CFs}$ were incubated with different concentrations of JSDS and Ang II $(1 \mu \mathrm{M})$ for 24 and $48 \mathrm{~h}$. (Fig. 2A and $\mathrm{B})$. The numbers of CFs were evaluated via cell count analysis, represented as an optical density value, and the content of hydroxyproline. Ang II significantly increased the number of CFs and the hydroxyproline concentration in the medium. JSDS effectively protected against the Ang II-induced proliferation of $\mathrm{CFs}$ in a concentration-dependent manner (Fig. 3A and B). Compared with the control group, the proliferation and collagen content of the Ang II-treated group were significantly increased $(\mathrm{P}<0.05)$. In the 10\% JSDS-treated group and 5\% JSDS-treated group, the increases in proliferation and collagen content were significantly decreased, compared with the Ang II-treated group $(\mathrm{P}<0.05)$. No significant alterations in the cell numbers or hydroxyproline levels were detected following JSDS treatment without Ang II stimulation (data not shown).

JSDS suppresses Ang II-induced collagen synthesis in CFs. Previous studies have shown that CFs may promote the development of fibrosis by producing fibrosis-associated factors, including collagen I and III (29-31). The enhancement of collagen I and III represent the predominant phenotype in cardiac fibrosis. Therefore, the present study examined whether JSDS suppressed collagen synthesis following Ang II stimulation. Compared with the control group, Ang II significantly increased the mRNA expression of collagen I. The mRNA expression of collagen I was downregulated following pre-treatment with JSDS (Fig. 4A). Immunofluorescence staining showed that JSDS inhibited the protein expression of collagen I in the CFs (Fig. 4B and C). Taken together, these findings indicated that the CFs treated with Ang II had enhanced secretory effects and that JSDS may prevent the increased protein expression of collagen I.

As CFs can transform into myofibroblasts (MFs), the present study determined whether JSDS can affect the phenotypic switching of CFs following treatment with Ang II. The mRNA and protein expression levels of $\alpha$-SMA were detected using RT-qPCR analyses and immunofluorescence. The results showed that the expression of $\alpha-S M A$ was significantly increased following Ang II stimulation at the mRNA and protein levels, which were decreased following JSDS treatment (Fig. 5A-C).

JSDS suppresses Ang II-induced TGF- $\beta 1$ and $\mathrm{p}$-Smad 2/3. Following stimulation with Ang II, the mRNA levels of 
A

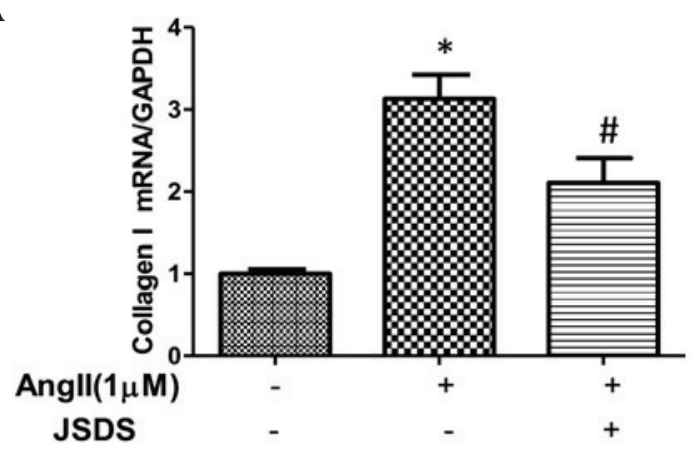

B

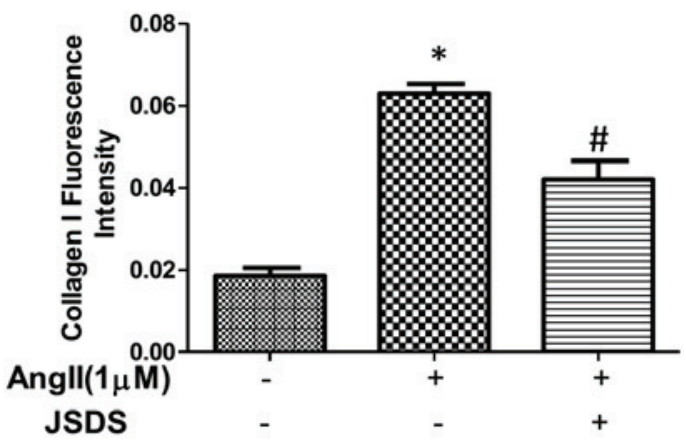

C
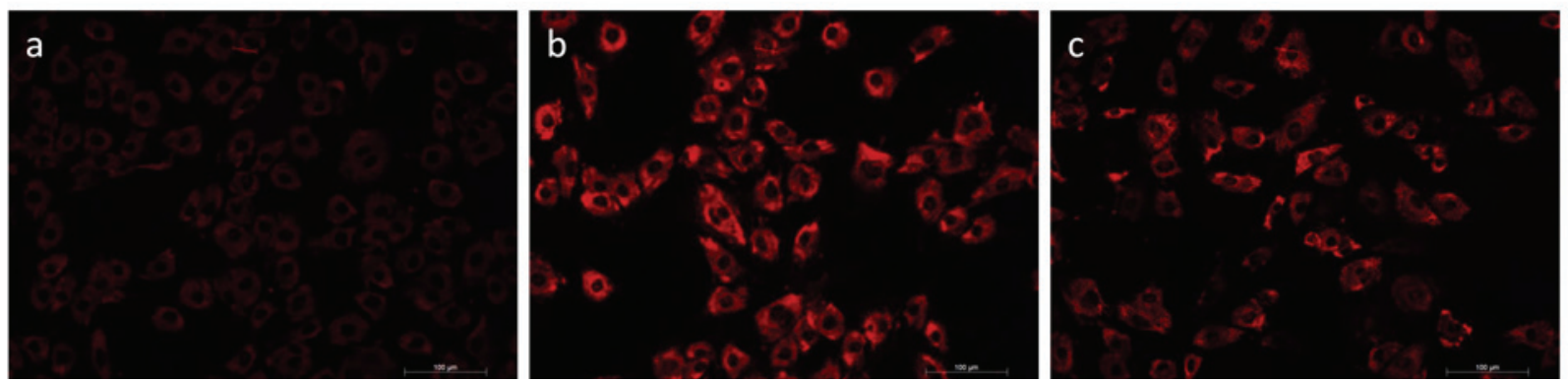

Figure 4. Effects of JSDS on Ang II-induced expression of collagen I in CFs. (A) mRNA expression levels of collagen I were assessed using reverse transcription-quantitative polymerase chain reaction analysis. (B) Fluorescence intensity of the expression of collagen I. (C) Cellular collagen I was visualized using fluorescein isothiocyanate-conjugated IgG in immunofluorescence staining. Representative images of the (a) control group, (b) Ang II-treated group. and (c) JSDS-treated group. Values are expressed as the mean \pm standard error of the mean $(\mathrm{n}=3)$. ${ }^{*} \mathrm{P}<0.05$, compared with the control group; ${ }^{\text {}} \mathrm{P}<0.05$, compared with the Ang II-treated group. Original magnification, x20, with representative images shown; $n=3$. CFs, cardiac fibroblasts; JSDS, Jia-Shen decoction-medicated serum; Ang II, angiotensin II.

A

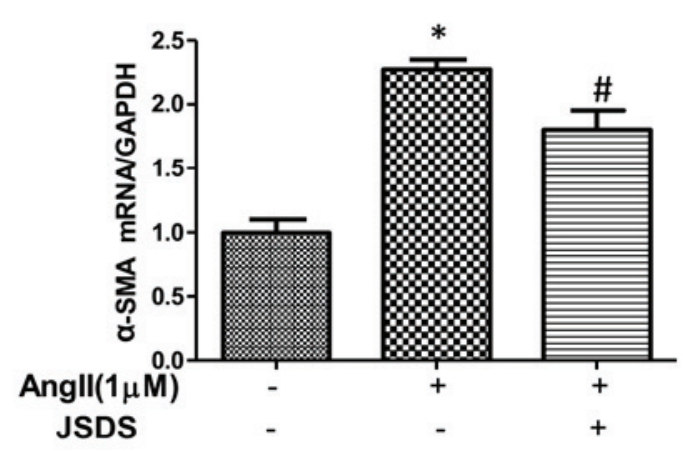

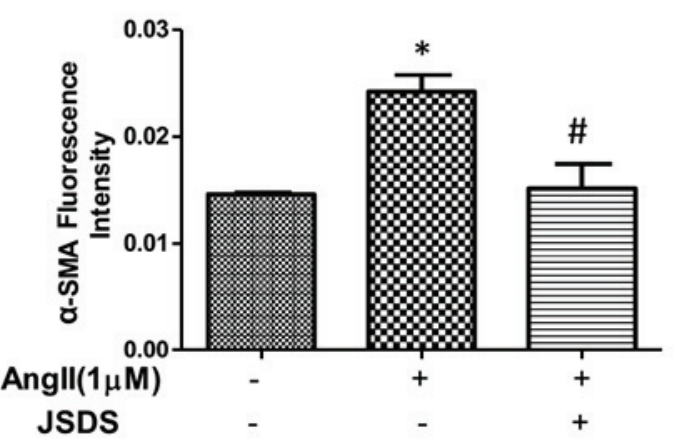

C
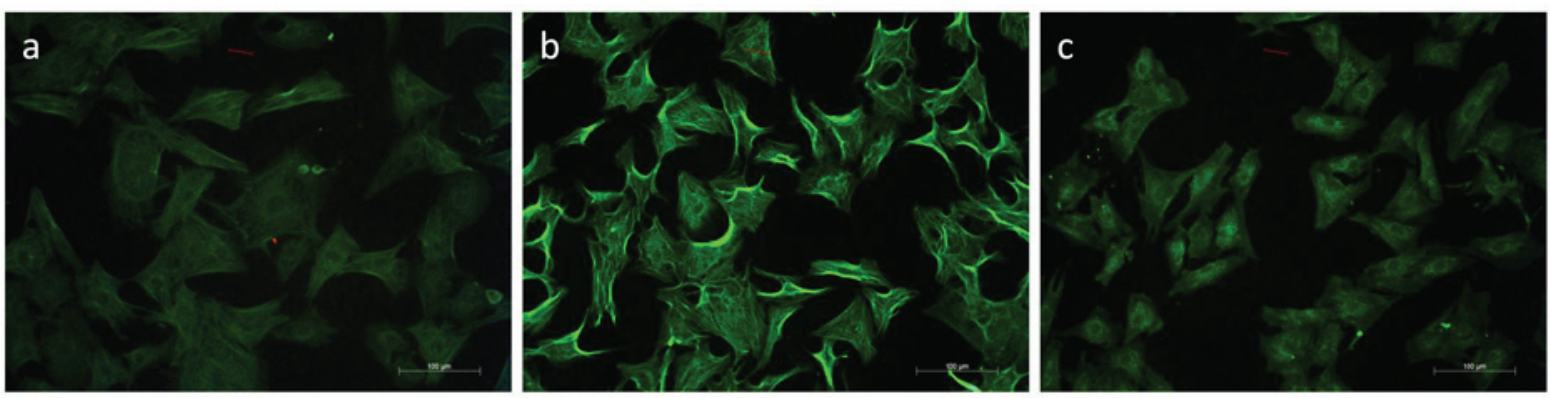

Figure 5. Effects of JSDS on Ang II-induced expression of $\alpha$-SMA in CFs. (A) mRNA expression levels of $\alpha$-SMA in the different groups were assessed using reverse trancsription-quantitative polymerase chain reaction analysis. (B) Fluorescence intensity of the expression of $\alpha$-SMA. (C) Cellular $\alpha$-SMA was visualized using fluorescein isothiocyanateconjugated $\operatorname{IgG}$ by immunofluorescence staining. Representative images of the (a) control group, the (b) AngII-treated group and the (c) JSDS-treated group. Values are expressed as the mean \pm standard error of the mean $(n=3) .{ }^{*} \mathrm{P}<0.05$, compared with the control group; ${ }^{\text {"}} \mathrm{P}<0.05$, compared with the Ang II-treated group. Original magnification, $\mathrm{x} 20$, with representative images shown; $\mathrm{n}=3$. CFs, cardiac fibroblasts; JSDS, Jia-Shen decoction-medicated serum; $\alpha$-SMA, $\alpha$-smooth muscle actin; Ang II, angiotensin II. 
A

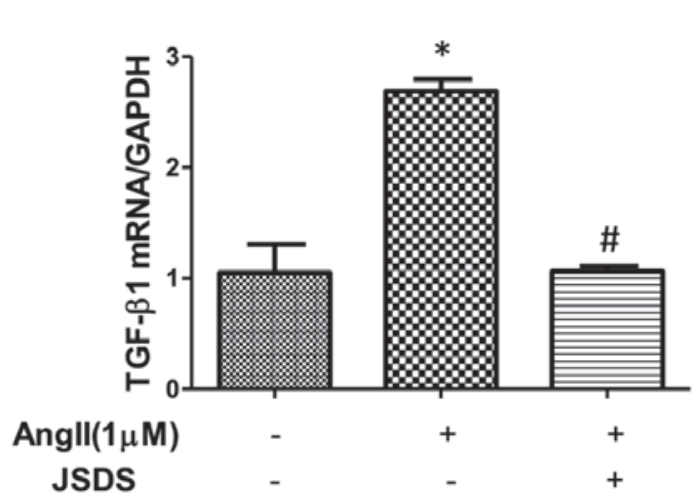

B TGF- $\beta 1$

\section{GAPDH}

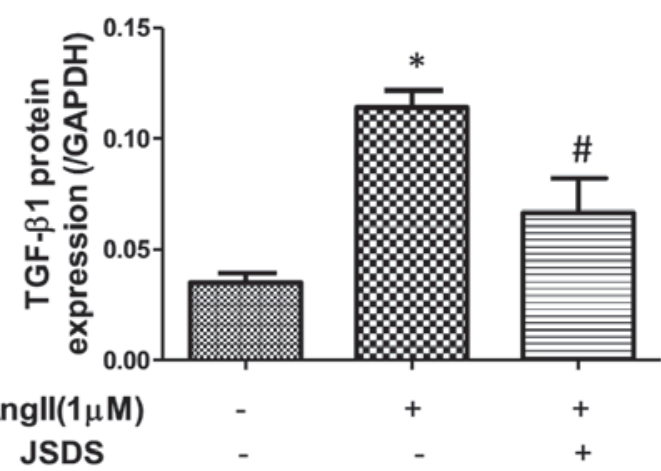

Figure 6. JSDS inhibits Ang II-induced relative mRNA and protein levels of TGF- $\beta 1$ in CFs. (A) mRNA expression levels of TGF- $\beta 1$ were assessed using reverse transcription-quantitative polymerase chain reaction analysis. Data were normalized to GAPDH mRNA. (B) Cell lysates were analyzed to determine the protein expression levels of TGF- $\beta 1$ using western blot analysis. Values are expressed as the mean \pm standard error of the mean ( $n=3$ ). $P<0.05$, compared with the control group; ${ }^{\#} \mathrm{P}<0.05$, compared with the Ang II-treated group. CFs, cardiac fibroblasts; JSDS, Jia-Shen decoction-medicated serum; TGF- $\beta 1$, transforming growth factor- $\beta 1$; Ang II, angiotensin II.

A
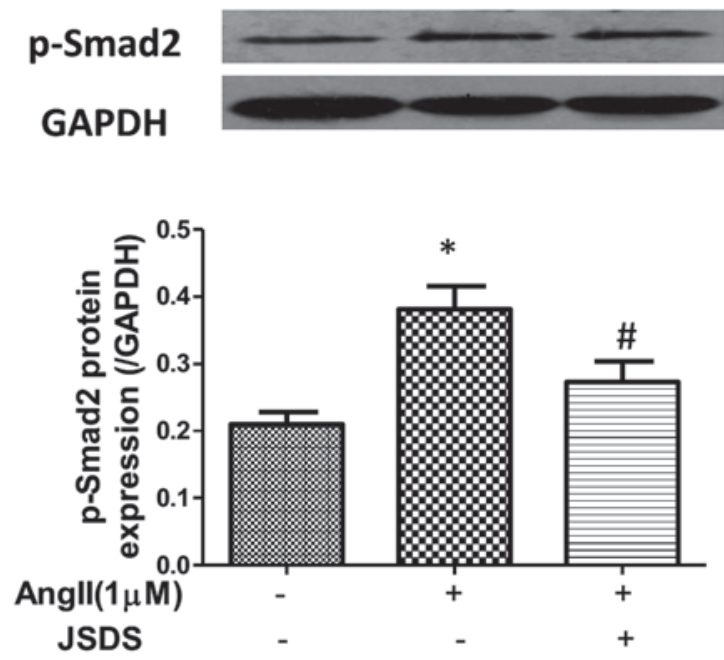

B

$$
\text { p-Smad3 }
$$

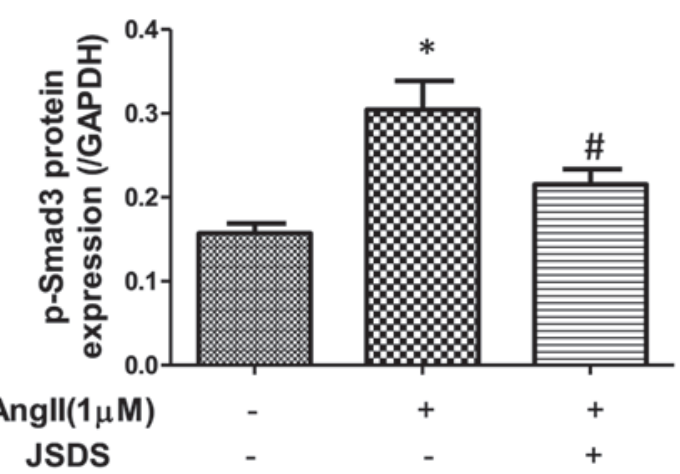

Figure 7. JSDS inhibits Ang II-induced expression of p-Smad2 and p-Smad3 in CFs. (A) Protein expression levels of p-Smad 2 were determined using western blot analysis. (B) Protein expression levels of p-Smad3 were determined using western blot analysis. Values are expressed as the mean \pm standard error of the mean $(\mathrm{n}=3)$. ${ }^{*} \mathrm{P}<0.05$, compared with the control group; ${ }^{*} \mathrm{P}<0.05$, compared with the Ang II-treated group. CFs, cardiac fibroblasts; JSDS, Jia-Shen decoction-medicated serum; p-Smad, phosphorylated small mothers against decapentaplegic; Ang II, angiotensin II.

$T G F-\beta 1$ in the CFs were significantly increased $(\mathrm{P}<0.05)$. The administration of JSDS prevented this by downregulating the mRNA level of $T G F-\beta 1$ stimulated by Ang II $(\mathrm{P}<0.05$; Fig. 6A). The protein levels of TGF- $\beta 1$ and $p-\operatorname{Smad} 2 / 3$ were significantly increased when the cells were treated with Ang II. The administration of JSDS prevented this, downregulating the protein levels of TGF- $\beta 1$ and $\mathrm{p}-\mathrm{Smad} 2 / 3$ stimulated by Ang II $(\mathrm{P}<0.05$; Figs. 6B, 7A and $\mathrm{B})$.

\section{Discussion}

Ang II is one of the most important renin-angiotensin system components, and is critical in cardiac remodeling (32). MFs are involved in reparative fibrosis in the infarcted heart. The differentiation of fibroblasts to MFs can be identified by certain features associated with the cytoskeleton, particularly by the expression of $\alpha$-SMA (33), which can synthesize collagen and generate a variety of fibrogenic factors, including TGF- $\beta 1$ and TNF- $\alpha$.

In the present study, it was found that Ang II stimulation increased cell proliferation and the collagen content of the cells, particularly collagen I. In addition, Ang II stimulation enhanced the mRNA and protein expression levels of $\alpha-S M A$ in the CFs, indicating the differentiation of CFs into MFs. Notably, the present study found that JSDS attenuated the collagen content and mRNA expression of $\alpha-S M A$, and decreased the generation of type I collagen, confirming its potential therapeutic value in MF treatment.

TGF- $\beta 1$ is one of the fibrogenic factors, which can induce the differentiation of CFs, and stimulate the synthesis of 
collagen and fiber connection protein. Smad proteins are molecules located downstream of the TGF- $\beta 1$ signal transduction pathway. Smad2 and Smad3 can be phosphorylated and activated through forming $\mathrm{p}-\mathrm{Smad} 2$ and $\mathrm{p}-\mathrm{Smad} 3$. The latter becomes a transcription complex by further combining with Co-Smads, following which it moves into the nucleus and completes the process of intracellular signal transduction. In the present study, western blot analysis showed that JSDS inhibited the protein expression levels of TGF- $\beta 1$, $\mathrm{p}-\mathrm{Smad} 2$ and $\mathrm{p}-\mathrm{Smad} 3$. In the control group, JSDS may affect in the Smad signaling pathways, which were activated in the process of myocardial fibrosis following treatment with Ang II.

In conclusion, the present study showed that JSDS inhibited proliferation, and decreased the expression levels of type I and type III collagen. These results clearly demonstrated that JSDS had a potential antifibrotic role, supporting the hypothesis that JSDS can reverse CF transdifferentiation by suppressing the expression of downstream targets, including TGF- $\beta 1$, p-Smad2 and $\mathrm{p}-\mathrm{Smad} 3$. These findings provide a novel perspective in understanding the molecular mechanism of the effects of JSDS on CF transdifferentiation and cardiac fibrosis.

\section{Acknowledgements}

This study was supported by a grant from the National Natural Science Foundation of China (grant no. 81173410).

\section{References}

1. Wynn TA and Ramalingam TR: Mechanisms of fibrosis: Therapeutic translation for fibrotic disease. Nat Med 18: 1028-1040, 2012.

2. Crabos M, Roth M, Hahn AW and Erne P: Characterization of angiotensin II receptors in cultured adult rat cardiac fibroblasts. Coupling to signaling systems and gene expression. J Clin Invest 93: 2372-2378, 1994

3. Dostal DE, Booz GW and Baker KM: Angiotensin II signalling pathways in cardiac fibroblasts: Conventional versus novel mechanisms in mediating cardiac growth and function. Mol Cell Biochem 157: 15-21, 1996.

4. Sadoshima J and Izumo S: Molecular characterization of angiotensin II-induced hypertrophy of cardiac myocytes and hyperplasia of cardiac fibroblasts. Critical role of the AT1 receptor subtype. Circ Res 73: 413-423, 1993.

5. Zhou G, Kandala JC, Tyagi SC, Katwa LC and Weber KT: Effects of angiotensin II and aldosterone on collagen gene expression and protein turnover in cardiac fibroblasts. Mol Cell Biochem 154: 171-178, 1996.

6. Schorb W, Booz GW, Dostal DE, Conrad KM, Chang KC and Baker KM: Angiotensin II is mitogenic in neonatal rat cardiac fibroblasts. Circ Res 72: 1245-1254, 1993.

7. Jia Y, Xu J, Yu Y, Guo J, Liu P, Chen S and Jiang J: Nifedipine inhibits angiotensin II-induced cardiac fibrosis via downregulating Nox4-derived ROS generation and suppressing ERK1/2, JNK signaling pathways. Pharmazie 68: 435-441, 2013.

8. Sassoli C, Chellini F, Pini A, Tani A, Nistri S, Nosi D, Zecchi-Orlandini S, Bani D and Formigli L: Relaxin prevents cardiac fibroblast-myofibroblast transition via notch-1-mediated inhibition of TGF-beta/Smad3 signaling. PLoS One 8: e63896, 2013.

9. Foo RS, Siow RC, Brown MJ and Bennett MR: Heme oxygenase-1 gene transfer inhibits angiotensin II-mediated rat cardiac myocyte apoptosis but not hypertrophy. J Cell Physiol 209: 1-7, 2006.

10. Kajstura J, Cigola E, Malhotra A, Li P, Cheng W, Meggs LG and Anversa P: Angiotensin II induces apoptosis of adult ventricular myocytes in vitro. J Mol Cell Cardiol 29: 859-870, 1997.

11. Choudhary R, Baker KM and Pan J: All-trans retinoic acid prevents angiotensin II- and mechanical stretch-induced reactive oxygen species generation and cardiomyocyte apoptosis. J Cell Physiol 215: 172-181, 2008.
12. Graiani G, Lagrasta C, Migliaccio E, Spillmann F, Meloni M, Madeddu P, Quaini F, Padura IM, Lanfrancone L, Pelicci P and Emanueli C: Genetic deletion of the p66Shc adaptor protein protects from angiotensin II-induced myocardial damage. Hypertension 46: 433-440, 2005.

13. Pan W, Zhong Y, Cheng C, Liu B, Wang L, Li A, Xiong L and Liu S: MiR-30-regulated autophagy mediates angiotensin II-induced myocardial hypertrophy. PLoS One 8: e53950, 2013.

14. Yang C, Liu Z, Liu K and Yang P: Mechanisms of Ghrelin anti-heart failure: Inhibition of Ang II-induced cardiomyocyte apoptosis by down-regulating AT1R expression. PLoS One 9: e85785, 2014.

15. Liu K, Deng X, Gong L, Chen X, Wang S, Chen H, Chen X, Amrit B and He S: The effect of intermedin on angiotensin II and endothelin-1 induced ventricular myocyte hypertrophy in neonatal rat. Clin Lab 59: 589-596, 2013.

16. Rosenkranz S: TGF-beta1 and angiotensin networking in cardiac remodeling. Cardiovasc Res 63: 423-432, 2004.

17. Zhu MJ, Wang YP, Xie SY, Liu WH, Li B, Wang YX, Wang H and Zhang BL: Protective effects of Jiashen Prescription () on myocardial infarction in rats. Chin J Integr Med 21: 417-422, 2015.

18. Yin D, Liu Z, Peng D, Yang Y, Gao X, Xu F and Han L: Serum containing Tao-Hong-Si-Wu decoction induces human endothelial cell VEGF production via PI3K/Akt-eNOS signaling. Evid Based Complement Alternat Med 2013: 195158, 2013.

19. Hong HJ, Liu JC, Cheng TH and Chan P: Tanshinone IIA attenuates angiotensin II-induced apoptosis via Akt pathway in neonatal rat cardiomyocytes. Acta Pharmacol Sin 31: 1569-1575, 2010.

20. Chen R, Xue J and Xie M: Osthole regulates TGF- $\beta 1$ and MMP-2/9 expressions via activation of PPAR $\alpha / \gamma$ in cultured mouse cardiac fibroblasts stimulated with angiotensin II. J Pharm Pharm Sci 16: 732-741, 2013.

21. Zou Y, Lin L, Ye Y, Wei J, Zhou N, Liang Y, Gong H, Li L, Wu J, Li Y, et al: Qiliqiangxin inhibits the development of cardiac hypertrophy, remodeling, and dysfunction during 4 weeks of pressure overload in mice. J Cardiovasc Pharmacol 59: 268-280, 2012.

22. Zhou J, Jiang K, Ding X, Fu M, Wang S, Zhu L, He T, Wang J, Sun A, Hu K, et al: Qiliqiangxin inhibits angiotensin II-induced transdifferentiation of rat cardiac fibroblasts through suppressing interleukin-6. J Cell Mol Med 19: 1114-1121, 2015.

23. Guo ZJ, Gao XM, Wang BH, Xu Q, Wang Y and Liu YP. Jiashenfang's effect to the nerve-internal secretion of CHF rats. J Liaoning Univ Tradit Chin Med (Chin) 11: 196-197, 2009.

24. Schröder D, Heger J, Piper HM and Euler G: Angiotensin II stimulates apoptosis via TGF-betal signaling in ventricular cardiomyocytes of rat. J Mol Med (Berl) 84: 975-983, 2006

25. Tao X, Fan J, Kao G, Zhang X, Su L, Yin Y and Zrenner B: Angiotensin-(1-7) attenuates angiotensin II-induced signalling associated with activation of a tyrosine phosphatase in Sprague-Dawley rats cardiac fibroblasts. Biol Cell 106: 182-192, 2014.

26. Cheng TH, Shih NL, Chen SY, Wang DL and Chen JJ: Reactive oxygen species modulate endothelin-I-induced c-fos gene expression in cardiomyocytes. Cardiovasc Res 41: 654-662, 1999.

27. Livak KJ and Schmittgen TD: Analysis of relative gene expression data using real-time quantitative PCR and the 2(-Delta Delta C(T)) Method. Methods 25: 402-408, 2001.

28. Zhang H, Wu J, Dong H, Khan SA, Chu ML and Tsuda T: Fibulin-2 deficiency attenuates angiotensin II-induced cardiac hypertrophy by reducing transforming growth factor- $\beta$ signalling. Clin Sci (Lond) 126: 275-288, 2014.

29. Dai B, Cui M, Zhu M, Su WL, Qiu MC and Zhang H: STAT1/3 and ERK1/2 synergistically regulate cardiac fibrosis induced by high glucose. Cell Physiol Biochem 32: 960-971, 2013.

30. Cao Y, Xu W and Xiong S: Adoptive transfer of regulatory T cells protects against Coxsackievirus B3-induced cardiac fibrosis. PLoS One 8: e74955, 2013.

31. Kazakov A, Hall R, Jagoda P, Bachelier K, Müller-Best P, Semenov A, Lammert F, Böhm M and Laufs U: Inhibition of endothelial nitric oxide synthase induces and enhances myocardial fibrosis. Cardiovasc Res 100: 211-221, 2013.

32. Souders CA, Bowers SL and Baudino TA: Cardiac fibroblast: The renaissance cell. Circ Res 105: 1164-1176, 2009.

33. Li P, Wang D, Lucas J, Oparil S, Xing D, Cao X, Novak L, Renfrow MB and Chen YF: Atrial natriuretic peptide inhibits transforming growth factor beta-induced Smad signaling and myofibroblast transformation in mouse cardiac fibroblasts. Circ Res 102: 185-192, 2008. 